\title{
COMBINATION OF TERRESTRIAL LASERSCANNING, UAV AND CLOSE-RANGE PHOTOGRAMMETRY FOR 3D RECONSTRUCTION OF COMPLEX CHURCHES IN GEORGIA
}

\author{
T. Luhmann ${ }^{1}$, M. Chizhova ${ }^{2}$, D. Gorkovchuk ${ }^{3}$, H. Hastedt ${ }^{1}$, N. Chachava ${ }^{4}$, N. Lekveishvili ${ }^{4}$ \\ ${ }^{1}$ Jade University, Institute for Applied Photogrammetry and Geoinformatics, Oldenburg, Germany - (luhmann)@jade-hs.de \\ ${ }^{2}$ University of Bamberg, Digital Technologies in Heritage Conservation - maria.chizhova@uni-bamberg.de \\ ${ }^{3}$ Kyiv National University of Construction and Architecture, Ukraine - denys.gorkovchuk@spm3d.com \\ ${ }^{4}$ Georgian Technical University, Faculty of Architecture, Urban Planning and Design, Tbilisi - n_chachava@gtu.ge
}

Commission II, WG II/8

KEY WORDS: Laserscanning, Photogrammetry, Data fusion, 3D reconstruction, Cultural heritage

\begin{abstract}
:
In September 2018, photogrammetric images and terrestrial laser scans were carried out as part of a measurement campaign for the three-dimensional recording of several historic churches in Tbilisi (Georgia). The aim was the complete spatial reconstruction with a spatial resolution and accuracy of approx. $1 \mathrm{~cm}$ under partly difficult external conditions, which required the use of different measurement techniques.

The local measurement data were collected by two laser scanning campaigns (Leica BLK360 and Faro Focus 3D X330), two UAV flights and two terrestrial image sets. The photogrammetric point clouds were calculated with the SfM programs AgiSoft PhotoScan and RealityCapture taking into account the control points from the Faro laser scan. The mean residual errors from the registrations or photogrammetric evaluations are 4-12 $\mathrm{mm}$, depending on the selected software. The best completeness and quality of the resulting 3D model was achieved by using laserscan data and images simultaneously.
\end{abstract}

\section{INTRODUCTION}

\subsection{Motivation}

In September 2018 photogrammetric images and terrestrial laser scans were carried out as part of a measurement campaign for the three-dimensional recording of three historical churches in Georgia. Several project objectives were defined in advance:

- 3D reconstruction of the outer skin of the buildings;

- 3D-reconstruction of an exemplary interior space;

- Use and examination of the new Leica BLK360 scanner under practical conditions;

- Combination of UAV and terrestrial image acquisition with terrestrial laserscans;

- Use of data for future semantic segmentation studies;

- Use of the data for restoration work and tourist purposes.

As part of ongoing research work on model-based reconstruction of destroyed structures from point clouds of Russian Orthodox churches (Chizhova et al. 2016), Jade University in Oldenburg and the University of Bamberg collaborate with Georgian Technical University in Tbilisi. In a one-week measuring campaign, three churches with different measuring systems were recorded:

- Terrestrial laser scanner Faro Focus 3D X330

- Terrestrial laser scanner Leica BLK360

- UAV DJI Mavic Pro with FC $220 \mathrm{f}=4.7 \mathrm{~mm}$

- DSLR camera Canon EOS 200D, $\mathrm{f}=10-20 \mathrm{~mm}$

- DSLR camera Canon EOS D6 Mark II, f=24-50mm
The aim was the complete spatial reconstruction with a spatial resolution and accuracy of approx. $1 \mathrm{~cm}$ under partly difficult external conditions, which required the combination of different measuring techniques. The significance of the work was also in definition of epochal architecture connected with Russian empire expansion policy and influence to local life by orthodox rules which reflects in architecture with its positive and negative sides. First of all, individual point clouds are generated from the laser scans and the photogrammetric images. The photogrammetric evaluations are carried out with Agisoft PhotoScan and RealityCapture. In a subsequent fusion step, the point clouds are merged into an overall model.

A tachymetric control point measurement could not be carried out for technical reasons. It was therefore decided to take control points for photogrammetric orientation from the registered point clouds of the laser scanning.

Studies of a similar kind are available from the literature, but refer to other used hardware and software solutions. Examples include Adamopoulos et al. (2017), Kersten et al. (2015) and Remondino (2011). First results of this project have been published by Chizhova et al. (2019).

\subsection{Measurement objects}

Originally planned were recordings of the Georgian churches Lurdji and Sioni in the Georgian capital Tbilisi. Due to the spatial proximity of the Andreas Church in Lurdji, it was decided at short notice to also include the neighbouring Russian Orthodox Church of St. John. Descriptions of the objects can be found in Anchabadze \& Volkova (1990) and Beridze (2014).

\footnotetext{
* Corresponding author
} 
1.2.1 Lurdji Monastery: The Lurdji Monastery is an orthodox architectural complex in Tbilisi (Figure 1). The monastery was founded in the 7th century, the oldest parts date back to the 12th century, which corresponds to the reign of Queen Tamar. In the 16th century the monastery was destroyed by Persians and restored only a century later. Currently, the sacral complex consists of two churches and the associated historical park Vera. After its destruction, the Andreas Church was restored in the 17th century as a basilica with a three-part nave. At the end of the 19th century, the church was reconstructed according to a project by A. Chizhov and received a new spherical dome, which was not typical for Georgian architecture. This dome was replaced in 1995 by a classic Georgian conical dome. The roof, once covered with blue ceramic tiles, is now made of blue-grey painted zinc sheets. The roof design determined the name of the monastery: "lurdji" means blue. Due to the various alterations, the church is not in the typical style of Georgian churches with a cruciform ground plan, but in a rectangular basic form with a cross-roof and a central tower, which has a cylindrical base and a conical top.

The church measures approximately $17 \mathrm{~m}$ x $12 \mathrm{~m} \times 25 \mathrm{~m}$ (W x L $\mathrm{x} \mathrm{H}$ ). It is located in the middle of a built-up residential area and the park on the edge, so that access from the ground was sometimes very restricted. The material of the outer walls consists of sandstone or brick and has a natural roughness as well as broken out places, which resulted from weathering and wear. The roof surfaces are largely texturally poor.

For the object the measurement of the outer skin was determined. Two UAV flights, two terrestrial DSLR image sets and two laser scan measurements were conducted.
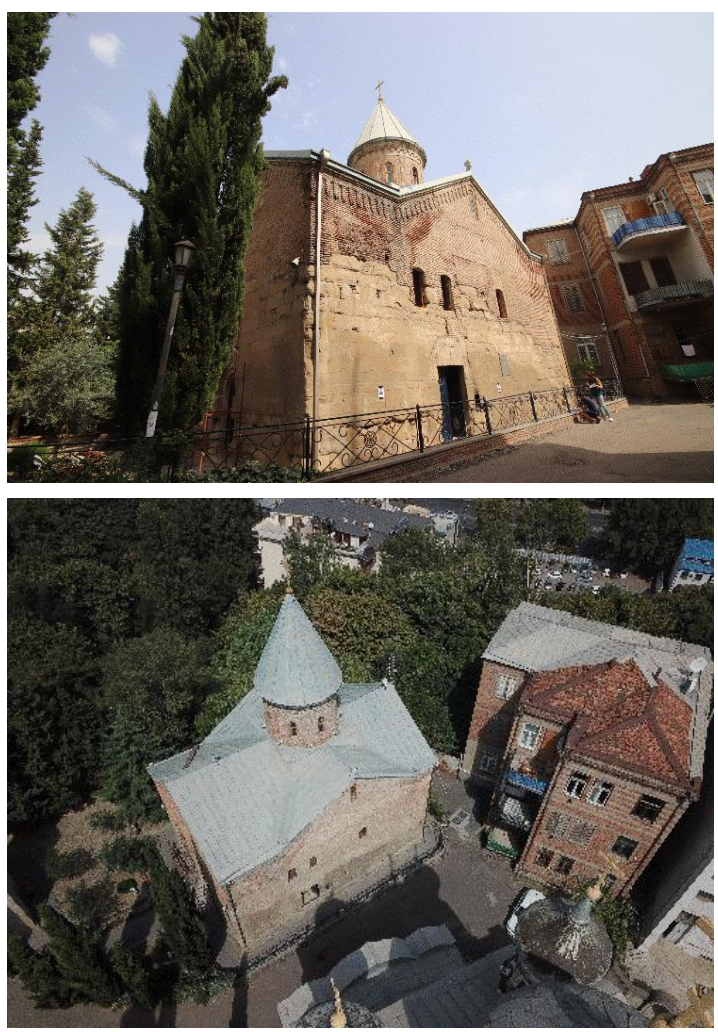

Figure 1. Andreas Church in Lurdji complex

1.2.2 Additional churches: During the measuring campaign, the church of St. John, which is also located in the Lurdji complex, was also recorded (Figure 2). It was built in 1898-
1901 by the Russian governor G. Golitsyn as a classical Russian church with a nave construction and five onion domes. Due to the considerable restrictions in accessibility, only a laser scan of this object was recorded with the Leica BLK360. A photogrammetric image could not be obtained.

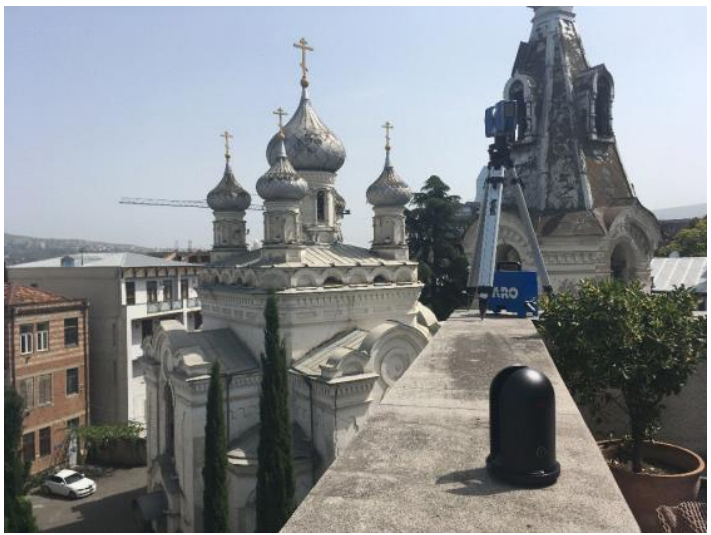

Figure 2. Russian-orthodox church in Lurdji complex; in foreground: Leica BLK360; in background: Faro scanner

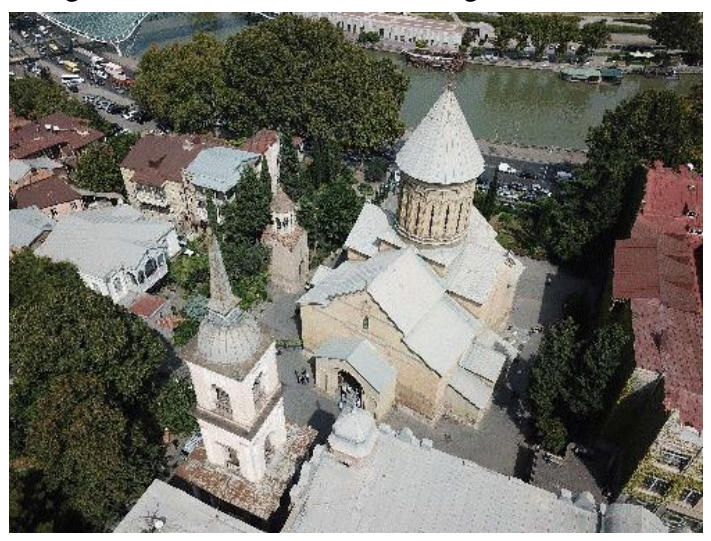

Figure 3. Sioni Cathedral

The Sioni Cathedral is the historical main church of the city of Tbilisi and one of the most important centres of Georgian orthodoxy (Figure 3). The construction of the original church began in the 6th century by the Georgian king Wachtang I. Gorgassali, its final construction dates back to the 7th century. This first church was completely destroyed after the establishment of the Tbilisi Emirate. The new Sioni Cathedral was built in the 12th century and underwent several destructions and reconstructions (17th and 18th centuries). After its restoration in 1980-1983, the church retained its medieval appearance and stylistically corresponds to the classical preMongolian religious architecture of Georgia.

The building was recorded both from the outside and from the inside. In total, two UAV flights, three terrestrial DSLR image sets and four laser scan measurements were carried out in the interior and exterior.

\section{LASER SCANNING}

\subsection{Targeting}

All recorded objects were marked with targets which were placed at a distance of approx. 5-10 $\mathrm{m}$ from each other on the building and in the rear surroundings (overview in Figure 4). As mentioned before, the targets could not be measured by 
totalstations, but were used for laser scanning exclusively as tie points, whose 3D coordinates were then used for photogrammetric datum definition.

The targets consisted of the usual chessboard-like signals (A4 size). In addition, some tilt $\&$ turn targets could be used, which are rotated around their own axis on tripods without changing the target centre, in order to be able to measure them optimally from different positions and from the air.

\subsection{Faro Focus 3D X330}

The Faro Focus 3D X330 (Figure 2 in the background) is a geodetic laser scanner with phase-based distance measurement and a 3D point accuracy of approx. $2 \mathrm{~mm}$ at $10 \mathrm{~m}$ distance. The maximum range is $330 \mathrm{~m}$. For laser scanning, a point distance of $6 \mathrm{~mm}$ at a distance of $10 \mathrm{~m}$ was selected. In this mode, a complete scan takes approx. 25 minutes, including the acquisition of image data. An overview of the captured data can be found in Table 1 .

\begin{tabular}{|l|c|c|}
\hline & Faro & BLK \\
\hline Stations & 11 & 20 \\
3D points (Mio.) & 350 & 784 \\
Scan duration (h) & 4.5 & 1.5 \\
Mean resolution (mm) & $3-5$ & $4-8$ \\
Reg. prec. Register (mm) & 6 & 9 \\
Reg. prec. Cyclone (mm) & 4 & 4 \\
\hline
\end{tabular}

Table 1. Laser scan data

In addition to 9 ground level positions, two scans were taken from elevated positions from the neighbouring bell tower and from a roof terrace of another building (Figure 2). Due to the limited choice of location, some parts of the roof and the tower cone could not be captured. The point resolution at the object varies between approx. $1 \mathrm{~mm}$ and $30 \mathrm{~mm}$ (tower) due to very different measuring distances.

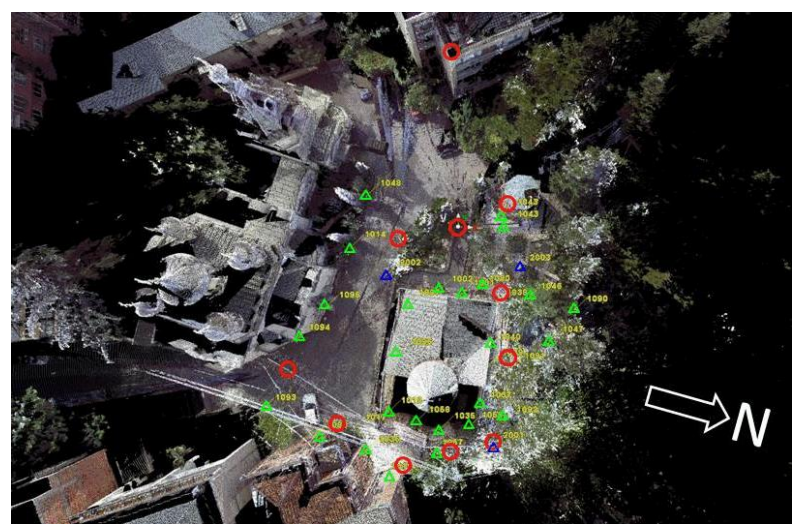

Figure 4. Overview of the registered Faro total point cloud with distribution of positions (red) and target marks (paper marks green, profit targets blue)

On site, the Faro Scene program was used to carry out an initial registration of all individual point clouds in order to check the quality and completeness of the data. Later, the data was registered with Register 360, which allows the extraction of 3D coordinates of the automatically measured targets. The registration was carried out via the displayed targets with subsequent optimization (ICP). The average standard deviation of the total registration is $6 \mathrm{~mm}$. Registration with the Cyclone software improves the precision of the registration to $4 \mathrm{~mm}$. The mean point resolution is between $3 \mathrm{~mm}$ in areas close to the ground and $5 \mathrm{~mm}$ on the tower. Figure 4 shows an overview of the registered point cloud with the positions and the recorded targets.

\subsection{Leica BLK360}

The Leica BLK360 laser scanner (Figure 2 in the foreground), which has been available on the market since 2017, is particularly easy to use. The device is primarily designed for simple building surveys with medium accuracy requirements at a range of up to $60 \mathrm{~m}$. Initial studies on performance (Blaskow et al., 2018) confirm the 3D point accuracy of approx. $6 \mathrm{~mm}$ at a distance of $10 \mathrm{~m}$ as specified by the manufacturer. The distance measurement is carried out according to the WFD principle (wave form digitizer). Laser scanning with the BLK was carried out in High Density mode, which corresponds to a point distance of $6 \mathrm{~mm}$ at $10 \mathrm{~m}$ distance. In this mode, a complete scan, including the acquisition of panorama images, takes approx. 5 minutes. An overview of the acquired data can be found in Table 1.

With BLK, the captured data is first stored in the scanner and then transferred to a tablet computer via WiFi.

Also for the BLK scans a first registration with the program ReCap was carried out on site. The later total registration with Register 360 resulted in an average standard deviation of $9 \mathrm{~mm}$. The registration was carried out using the targets with subsequent optimization (ICP). The mean point resolution lies between $4 \mathrm{~mm}$ in areas close to the ground and $8 \mathrm{~mm}$ at the tower. When registering with the Cyclone software, the precision of the registration improves to $4 \mathrm{~mm}$. Figure 5 shows an overview of the registered point cloud with the positions and the recorded targets.

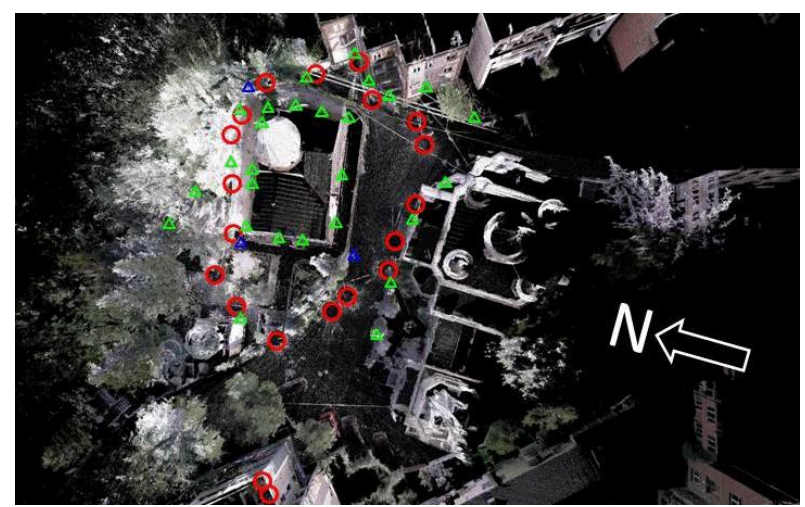

Figure 5. Overview of the registered BLK total point cloud with distribution of positions (red) and target marks (paper marks green, profit targets blue)

\subsection{Comparative analysis}

2.4.1 Registration: Both scanners could be used without any problems in a practical project. Although the BLK360 is more likely to be classified as an indoor scanner or for simple BIM applications with lower accuracy requirements, it has also proven to be a suitable scanner for complete building survey in this project. The fast and simple data acquisition is particularly advantageous compared to the Faro. Disadvantages are the very limited battery capacity and the missing USB interface or SD memory card for data transfer without network connection, as well as a lower robustness against sunlight and heat. Based on the technical specifications and the registration results, it can be 
assumed that the Faro point cloud is of better quality than that of the BLK.

Software-dependent deviations in the registrations, which are not due to data quality but to problems with data export and system settings, have not yet been finally clarified.

2.4.2 Quality of point clouds: The quality of the point clouds is analysed in this article only exemplarily. The deviations of the BLK scan from the Faro point cloud are examined as a reference. A first comparison of the two registered and unfiltered point clouds on an outer wall by cloudto-cloud comparison (function Cloud/Cloud-Distance in CloudCompare, see Figure 6 top) shows that both point clouds fit together in the range of $5-10 \mathrm{~mm}$, with the exception of the areas of the object edges (building corners, roof ridge). Straightline jumps in the deviations are conspicuous, which are presumably due to systematic residual deviations of the registrations or the inclusion of scans from neighbouring buildings. Furthermore, a growing discrepancy with increasing building height is clearly discernible. Their cause probably lies in the exclusively horizontal distribution of the target marks as well as possibly a worse scan accuracy with increasing vertical angle.

Figure 6 bottom shows the result after an additional adjustment of the scans via ICP. This largely eliminates the heightdependent systematics, which again indicates a datum problem during registration. The histogram of the deviations shows a maximum at $4 \mathrm{~mm}$, which indicates a total systematic shift of the point clouds to each other.

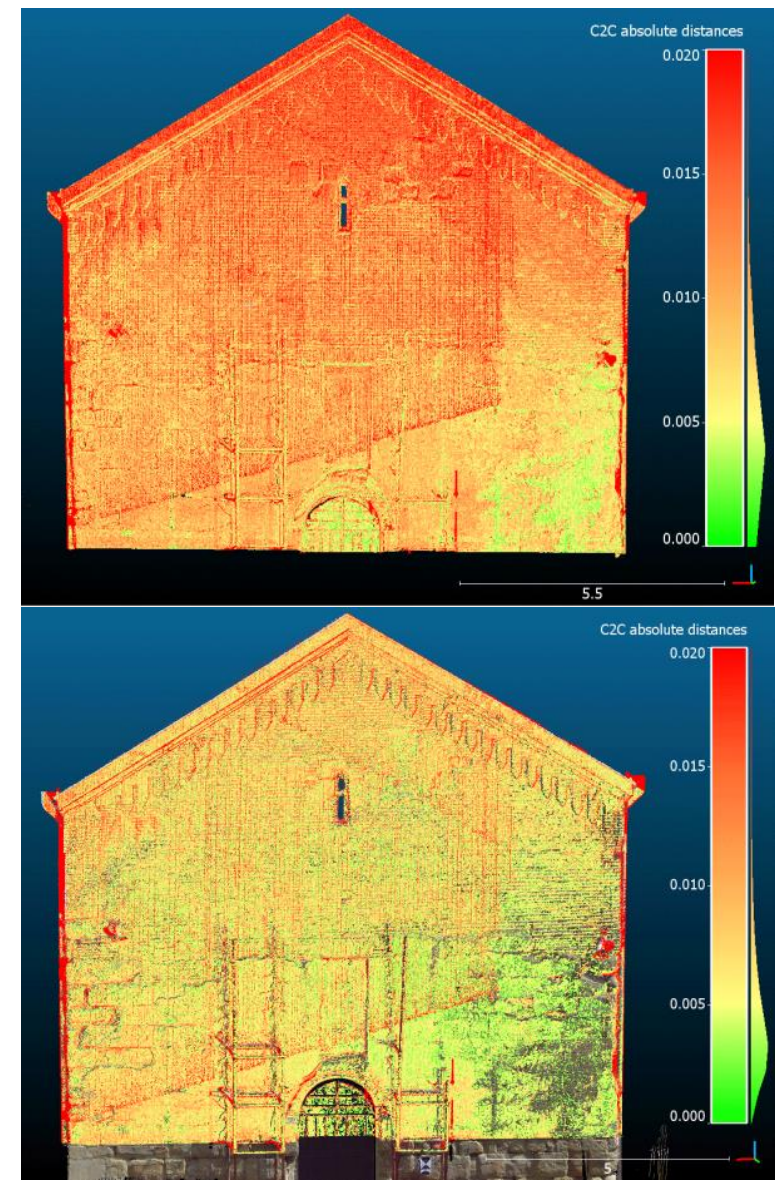

Figure 6. Comparison Faro-BLK: left Cloud-to-Cloud; right Cloud-to-Cloud after ICP (in mm)

\section{PHOTOGRAMMETRY}

\subsection{UAV flights}

The local partners provided a DJI Mavic Pro drone with FC 220 camera (12 Mpixel) (focal length $\mathrm{f}=4.7 \mathrm{~mm}$, image format $6.4 \mathrm{~mm} \times 4.8 \mathrm{~mm}$, pixel size $1.6 \mu \mathrm{m})$. The maximum flight duration is approx. 22 minutes. The drone was manually controlled by an experienced pilot. A meandering image configuration with vertical images as well as two circular flights with oblique images from different distances were captured. Table 2 compiles the most important flight data. Figure 7 shows example images of the three different flights.

\begin{tabular}{|l|c|c|c|c|c|}
\hline & Images & $\begin{array}{c}\text { Altitude } \\
(\mathrm{m})\end{array}$ & $\begin{array}{c}\text { Distance } \\
(\mathrm{m})\end{array}$ & $\begin{array}{c}\text { Image } \\
\text { scales }\end{array}$ & $\begin{array}{c}\text { GSD } \\
(\mathrm{mm})\end{array}$ \\
\hline Nadir & 173 & $30-35$ & $20-30$ & $4600-6500$ & $7-10$ \\
Circle 1 & 88 & $15-20$ & $14-25$ & $3600-5200$ & $5-9$ \\
Circle 2 & 107 & $45-55$ & $25-32$ & $5500-6700$ & $9-11$ \\
\hline Total & 368 & $15-55$ & $14-32$ & $3600-6700$ & $5-11$ \\
\hline
\end{tabular}

Table 2. UAV data
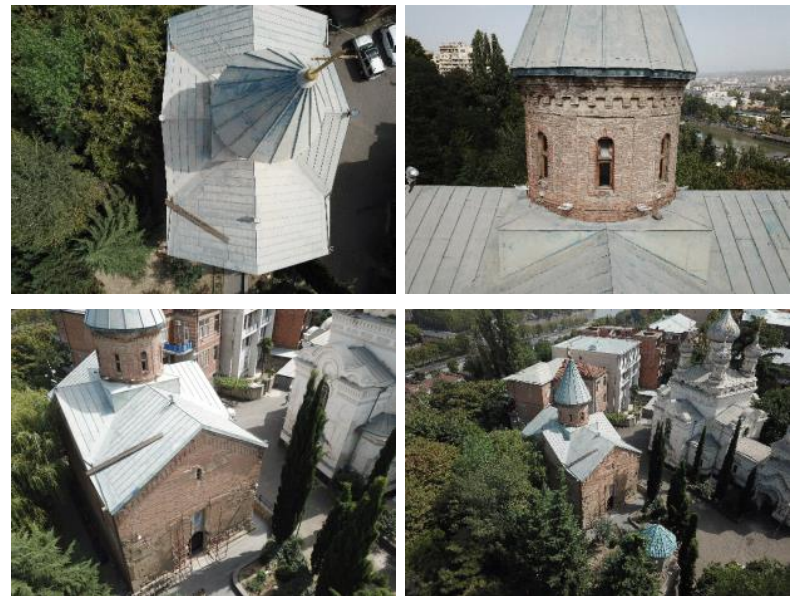

Figure 7. Example images of UAV flights

\subsection{Terrestrial images}

From the ground, two hand-held sets of images were taken with digital SLR cameras (DSLRs), each of which had zoom lenses since fixed-focus lenses were not available. The lenses were fixed in the shortest focal length with adhesive tape, autofocus and sensor cleaning were deactivated. The images were saved in JPEG format with low compression.

The camera Canon EOS D6 Mark II with lens $\mathrm{f}=24-50 \mathrm{~mm}$ has a 26.2 Mpixel full frame CMOS sensor $(35.9 \mathrm{~mm} \times 24.0 \mathrm{~mm}$, pixel size $6.5 \mu \mathrm{m})$ and an integrated GPS receiver. The Canon EOS 200D with lens $\mathrm{f}=10-20 \mathrm{~mm}$ has a 24.2 Mpixel CMOS sensor in DX format $(22.3 \mathrm{~mm} \times 14.9 \mathrm{~mm}$, pixel size $3.7 \mu \mathrm{m})$. The field angles related to the image diagonal are $84^{\circ}$ (Canon D6) or $106^{\circ}$ (Canon 200D).

The image acquisition was designed in such a way that the following criteria were largely met:

- High overlap for reliable measurement and matching of feature points;

- Combination of vertical and oblique images for optimum geometric intersection conditions; 
- Combination of close-up images (distance 1-2m) and long-distance images $(5-20 \mathrm{~m})$ to achieve a high object resolution with simultaneous large object coverage by overview images;

- Numerous tilted $\left(90^{\circ}\right.$ rotated) images for reliable camera calibration;

- Small aperture to achieve a sufficiently large depth of field.

The duration of the image acquisition was approx. 1.5 hours per image set.

\subsection{Processing}

3.3.1 Agisoft PhotoScan: The software program PhotoScan (new: MetaShape) of the Russian company Agisoft is a widely used Structure-from-Motion (SfM) program, which is used in many different fields of application. In addition to the orientation (alignment) and the adjusted object coordinates (sparse point cloud), it provides a dense point cloud generated by semi-global matching, surface meshing and true orthophotos. The software offers well documented calibration models for interior orientation and numerous import and export formats of all orientation parameters.

The automatic processing of all image data from the different data sets, which are subject to very different acquisition conditions and qualities for evaluation in PhotoScan, proved to be difficult. The automated alignment of all images referred to a clear orientation error, which was shown by the formation of two reconstructions of the church rotated towards each other. Figure 8 shows views of a reduced dense point cloud after the automatic orientation of all images. In addition to the double reconstruction of the same building, it can be seen here that these are strongly rotated (Figure 8 left) and that there is also a difference in scale (Figure 8 right). It could be identified that the images of the DJI and the Canon EOS 200D result in an equivalent reconstruction, while the images of the Canon EOS $6 \mathrm{D}$ lead to a clear misorientation. For this reason, in PhotoScan only a reduced image data set was used for the evaluation.
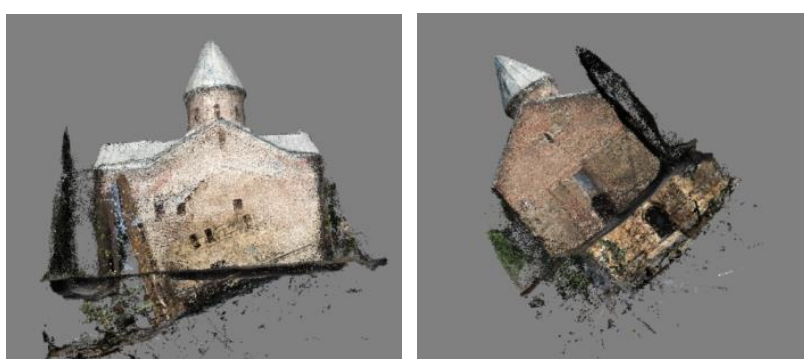

Figure 8. Views of a reduced dense point cloud after faulty automatic orientation in PhotoScan 1.4.1; left: faulty orientation with double reconstruction and rotation; right: front view of the

church with double reconstruction of the entrance portal
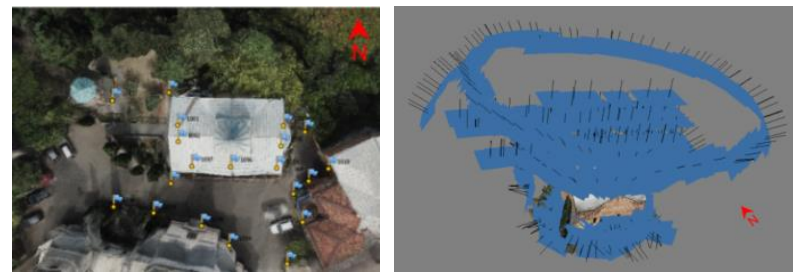

Figure 9. left: Control point overview of the evaluation in PhotoScan; right: Overview of the image data from image flight and terrestrial images after the image orientation in PhotoScan
The image data of the UAV flight and the Canon EOS 200D were evaluated in separate blocks (chunks). Each image data set of a camera was automatically oriented and checked in the resolution level "medium" (reduced by factor 4 to the original resolution). Only two images were eliminated in the automatic orientation process. Figure 9 right shows all image positions after merging the separate blocks. The control points were previously measured separately in the blocks. A total of 20 control points were used for georeferencing in the initial processing (see Figure 9 left). Due to the vegetation around the church, no control points were identified on the northern side of the church, as they are only shown in the terrestrial image material. Most of the northern facade under the foliage of the adjacent vegetation is covered for aerial photographs.

\begin{tabular}{|l|c|c|}
\hline \multicolumn{1}{|c|}{ Software } & PhotoScan & RealityCapture \\
\hline Aligned images & 572 & 1100 \\
$\mathrm{RMS}_{\text {back }}(\mathrm{px})$ & 0.9 & 0.6 \\
$\mathrm{RMS}_{\text {obj }}(\mathrm{mm})$ & 8 & 5 \\
Computing time & $1 \mathrm{~h}$ & $7 \mathrm{~min}$ \\
Alignment & & \\
\hline Computing time & $5 \mathrm{D}$ & $4.5 \mathrm{~h}$ \\
Dense pointcloud & & \\
\hline
\end{tabular}

Table 3. Photogrammetric processing

The combined blocks were then jointly adjusted taking into account the simultaneous calibration of the cameras involved (f, $\mathrm{cx}, \mathrm{cy}, \mathrm{k} 1-\mathrm{k} 3, \mathrm{p} 1-\mathrm{p} 2, \mathrm{~b} 1-\mathrm{b} 2)$. The average reprojection error $\left(\mathrm{RMS}_{\text {back }}\right)$ is 0.9 pixels for all tie points and 0.4 pixels for the control points. The mean $3 \mathrm{D}$ deviation at the control points is $8 \mathrm{~mm}$ with an a priori accuracy of $7 \mathrm{~mm}$ (see Table 3). The achieved deviations appear realistic. Nevertheless, the distributions of the image residuals in PhotoScan (Figure 10) for both cameras show a clear systematics, which either indicate deficiencies in the modelling of the interior orientation or are due to the implementation. RealityCapture does not provide any output of image residuals.
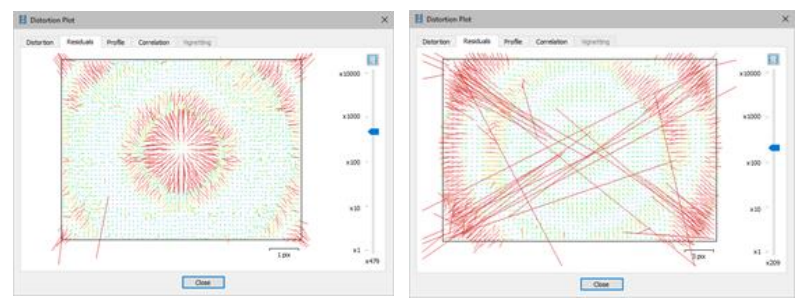

Figure 10. Image residuals after bundle adjustment in PhotoScan; left: DJI FC 220; right: Canon 200D

The textured and meshed 3D model from Figure 11 is based on the generation of a dense point cloud in the quality level "high" (reduced by factor 2 to the original resolution, 42 million points). The subsequent $3 \mathrm{D}$ meshing was carried out with the highest mesh density (resulting in approximately 8.4 million areas). For texturing, the "adaptive orthophoto" method was chosen, which selects the image on the basis of the surface alignment and at the same time allows mosaicing. 

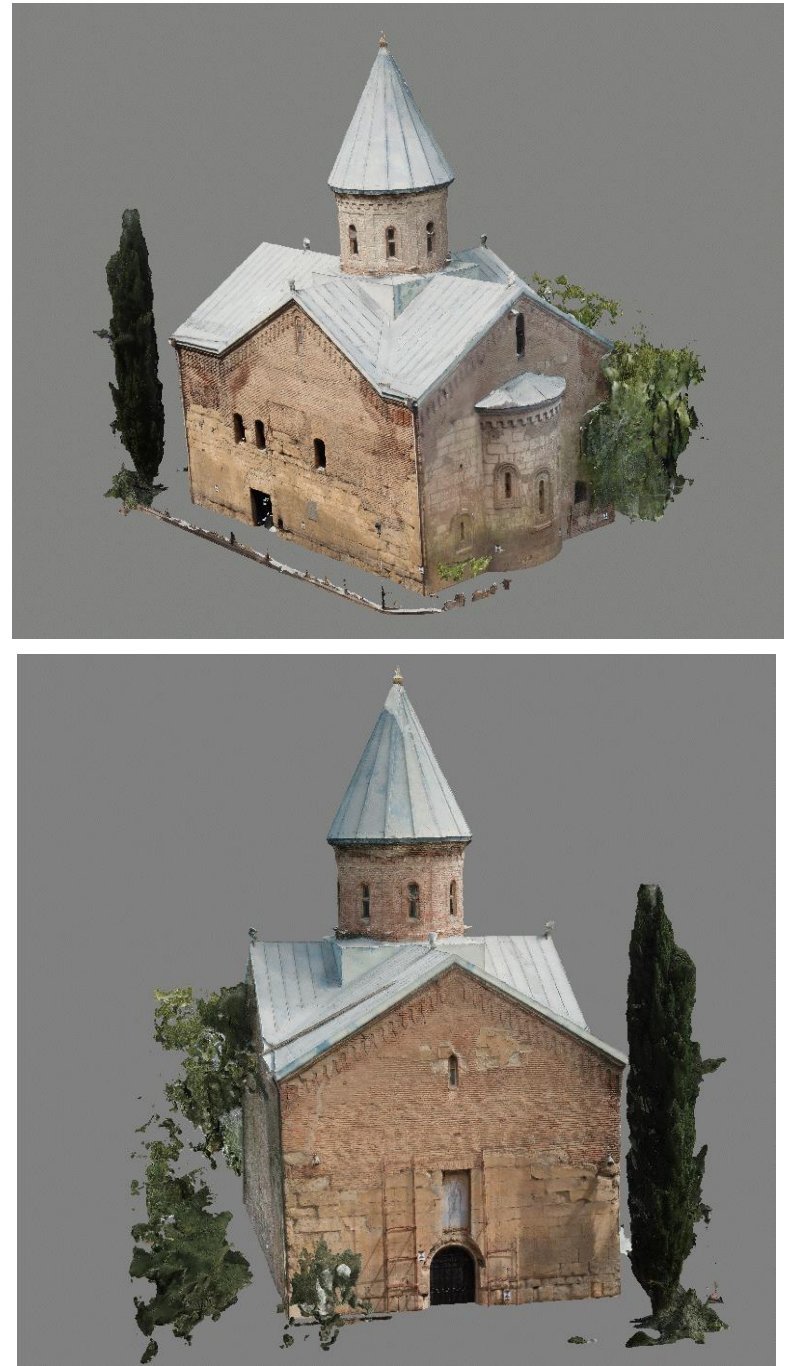

Figure 11. Textured and meshed 3D model from PhotoScan (Alignment "medium", Dense cloud "high", Mesh "arbitrary, high", Texture "adaptive orthophoto, mosaic")

Due to the complex image arrangement and the object geometry, high computing times in the range of approx. 1 hour for the alignment and approx. 5 days for the calculation of the dense point cloud result (quality level "high", processor i7-4770 with $3.4 \mathrm{GHz}$ CPU, Ge-Force GTC 645, 16 MB RAM). The 3D model also refers to uncertainties in the reconstruction of the northern side of the church (occlusions due to vegetation, difficult image connections) as well as on the western side (short side in Figure 11 left). The western church portal can still be reconstructed with sufficient quality in the dense point cloud, but on the one hand the meshing results in a misconstruction of the semi-circular extension and on the other hand the model textures are very blurred and pale. These deviations are to be examined more closely and, if necessary, alternative modelling tools are to be used. The generation of a dense point cloud with higher resolution could lead to a higher quality 3D model. These and further statistical investigations for simultaneous calibration and adjustment of the image associations will follow in further analysis steps.

3.3.2 RealityCapture: The software RealityCapture of the Slovakian company Capturing Reality is another photogrammetry program based on SfM, which is characterized by its fast highly parallel, GPU-based data processing and the possibility of direct integration of laser scanning point clouds. It is implicitly assumed that the laser scanning data is of higher accuracy than the photogrammetric evaluation, i.e. the orientation of the images and the calculated point cloud is adapted to the laser scanning reference. A disadvantage of the program is the limited possibility of influencing the parameter control and the very poor output of statistical parameters of the results. Depending on the model selected for camera modelling, the parameters of the interior orientation are determined separately for each image (image-variant interior orientation). Photogrammetric orientation and calibration data cannot be exported to other formats. RealityCapture does not allow the export of point clouds, but only the output of completely meshed surface models.
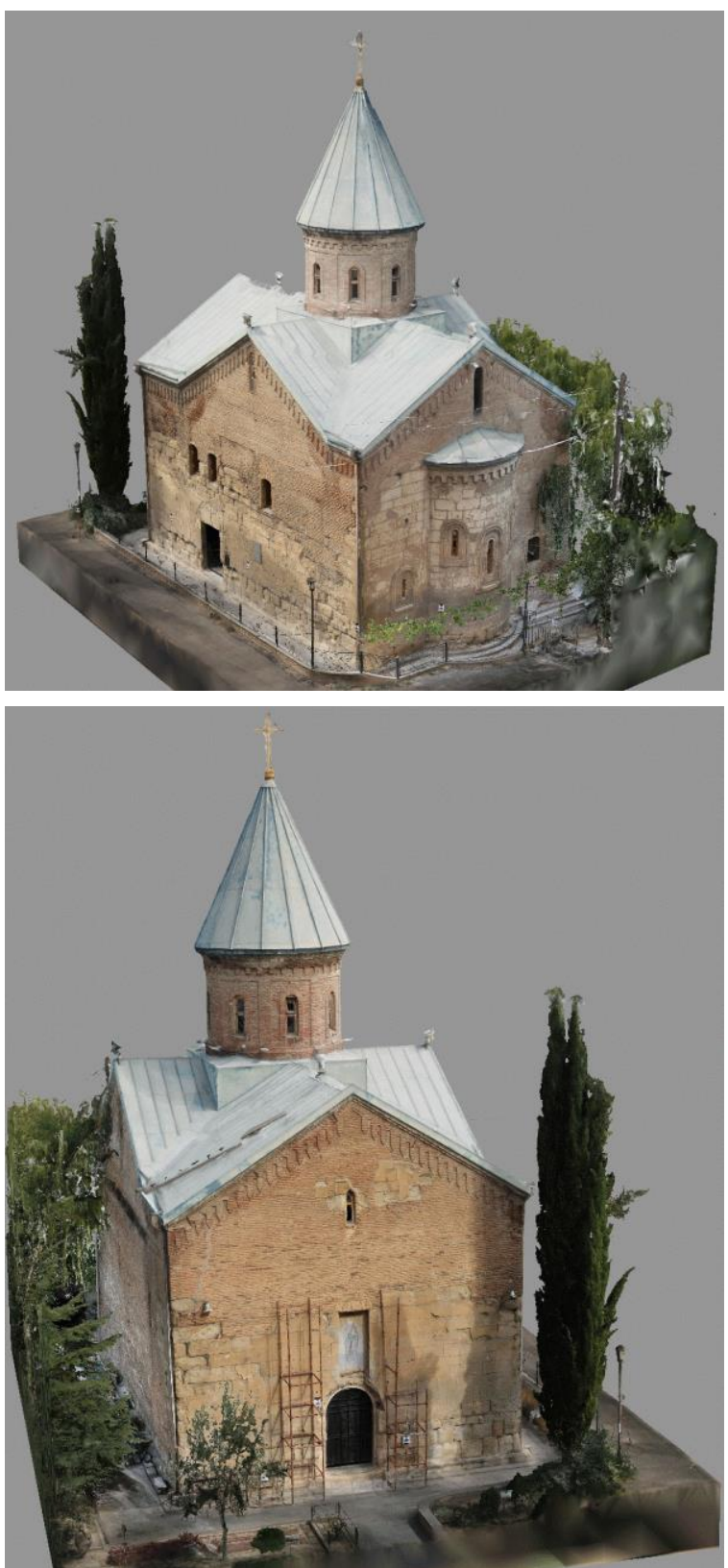

Figure 12. Meshed complete model with RealityCapture 1.0.3

In the Lurdji project all existing images of all cameras were evaluated together with the Faro point cloud including 22 control points. Thereby 1100 images of 1570 images were orientated fully automatically. Only in some areas of the roof 
and the spire the automatic image orientation did fail, as either predominantly homogeneous (textureless) object surfaces or strong variations between foreground (cross) and background (trees, park) occurred. For camera calibration a model with three radial-symmetric and two tangential distortion parameters was chosen. The mean reprojection error (Sigma 0) is 0.6 pixels.

The surface triangulated in RealityCapture consists of approx. 140 million triangles. The computing time for the alignment on a computer with i7 6700k processor, 64GB RAM and Nvidia GTX980TI graphics card was about 7 minutes, the calculation of the dense point cloud and meshing (quality level medium) took about 4.5 hours.

Figure 12 shows the calculated 3D model as meshing (approx. $5 \mathrm{~mm}$ point spacing). The high quality is proven on the one hand by the completeness of the overall model, but on the other hand detailed views also show how well the object was reconstructed, especially on the sides that are difficult to access (Figure 13). Finest details such as the scaffolding at the entrance or filigree cast-iron fences in the rear area are modelled correctly. Only the cross on the top of the tower still shows "shadows", which might be caused by the background problem mentioned above as well as some missing pictures.
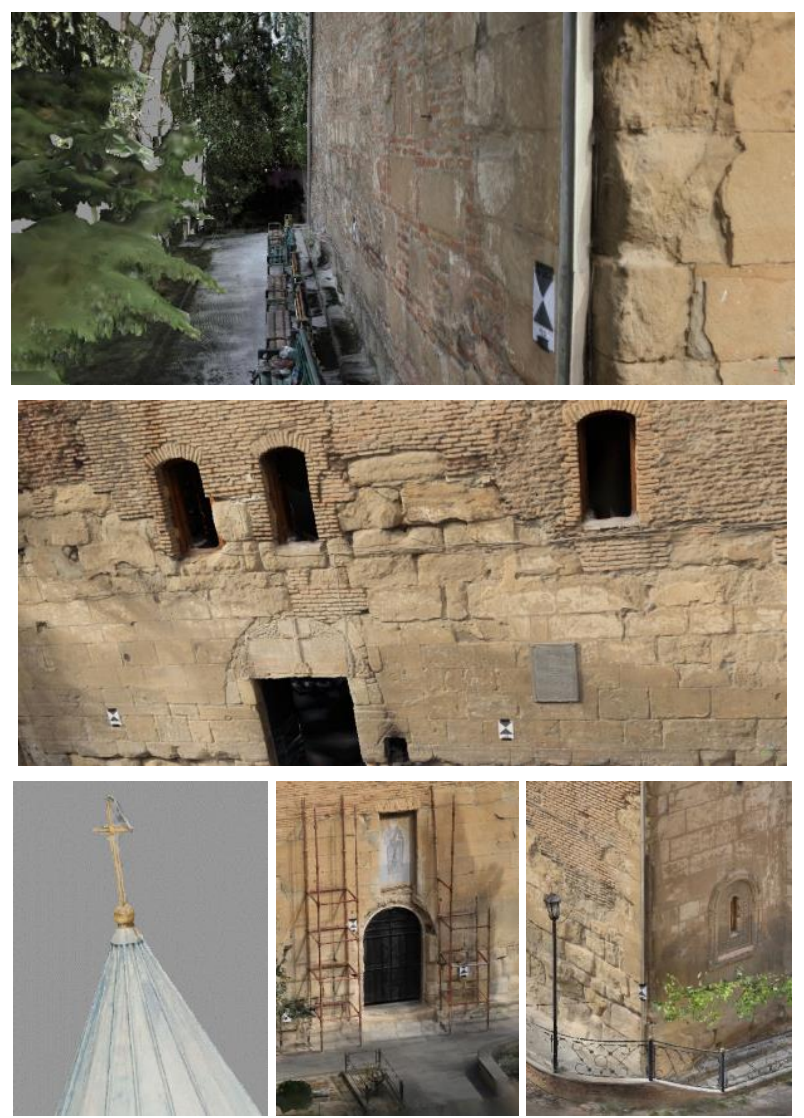

Figure 13. Detailed views from the point cloud calculated with RealityCapture

\subsection{Analysis of results}

The acquired images show different good configurations for SfM. While the UAV image flights can be easily oriented due to very high overlaps and uniform orientation data, this is only possible with manual intervention for the individual terrestrial image sets. In particular, the D6 data set shows some problem areas with too small overlaps or images falling out of the series that do not cover the object continuously in the sequence. Also problematic are images that are tilted by $90^{\circ}$, which can obviously disturb the orientation (alignment). If, however, all existing images are evaluated together, the orientation succeeds largely without problems except for a few, non-orientable images.

At the present time the result of RealityCapture is better than that of PhotoScan (Table 3). In addition, the computing times are considerably shorter. Due to the observed systematic residual errors during the camera calibration in PhotoScan as well as a not yet final analysis of the program settings and the control point configuration, these statements cannot yet be regarded as final. Altogether, the result of the photogrammetric evaluation shows that an average standard deviation of the 3D points of approx. $5 \mathrm{~mm}$ in object space is achievable. It should be noted that the control points were taken from the Faro scan and were not measured by totalstations (with possible higher accuracy).

The displayed point clouds are raw data without any filtering or manual post-processing. For subsequent applications or visualizations, however, they still have to be processed and cleaned. The exemplary comparison of both point clouds shows some serious differences in detail, which, however, cannot yet be finally evaluated. Since the Faro point cloud is integrated into the SfM process in RealityCapture, a point cloud is created that is very close to the laser scan result. However, the detailed analyses also show that e.g. filigree object details that are not resolved by the laser scanning are very well reconstructed by using the image information.

\section{FURTHER INVESTIGATIONS}

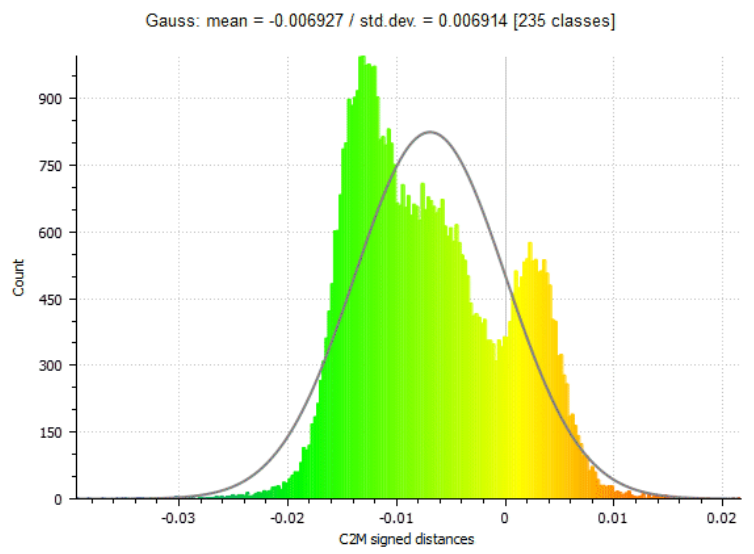

Figure 14 shows a first comparison (cloud-to-mesh) of the point cloud calculated with RealityCapture with all image data and simultaneous use of the BLK360 point cloud, compared to the 
reference from the Faro scan. Gauss: mean $=-0.006927 /$ std.dev. $=0.006914[235$ classes $]$

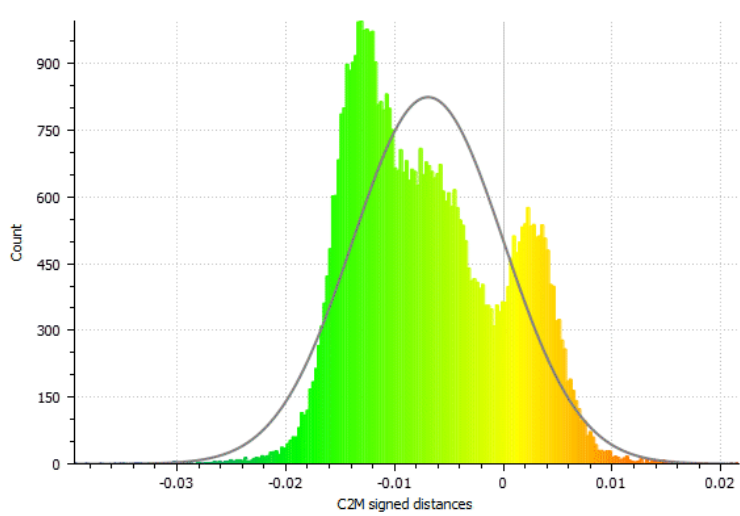

Figure 14a,b show the deviations over the entire object facade. The average deviation is only $2 \mathrm{~mm}$, i.e. the joint evaluation of the TLS and the image data leads again to a high-quality 3D model of the surface. Areas of similar deviations can be seen here, which can at least partly be explained by the very different surface texture of the masonry (see Figure 1). Gauss: mean $=-0.006927 /$ std.dev. $=0.006914$ [235 classes $]$

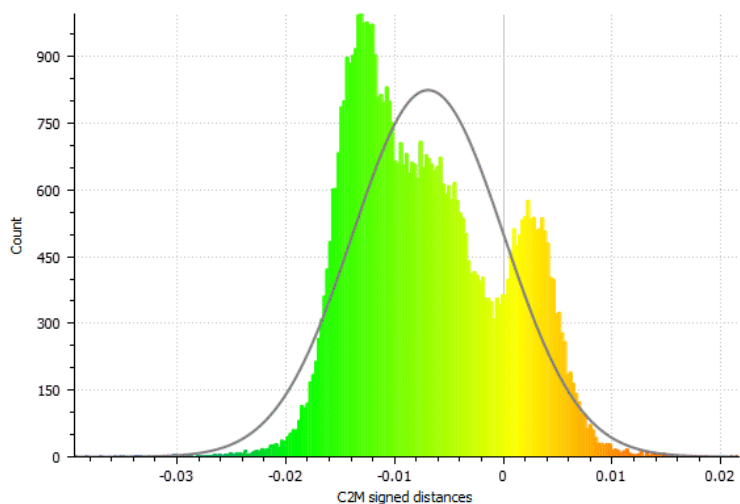

Figure $14 \mathrm{c}$ and $\mathrm{d}$ show the corresponding histograms of the deviations in the lower and upper areas of the façade. Here it becomes visible that in the upper range worse data are available, analogous to the result from section 2.4.2.

\section{SUMMARY}

This report presents the first results of an extensive measurement campaign carried out in Georgia in September 2018. The initial aim was to evaluate the use of different measurement systems and evaluation methods for the 3D reconstruction of historical churches under real conditions in order to gain experience for future projects. Basically, terrestrial laserscanning and photogrammetric methods based on structurefrom-motion provide similar results.

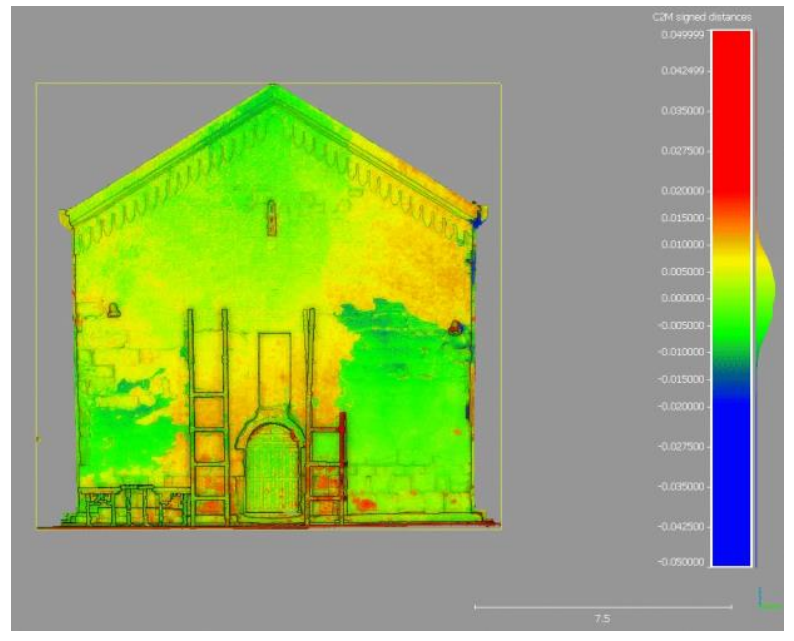

Gauss: mean $=-0.005746 /$ std.dev. $=0.002614[190$ classes $]$

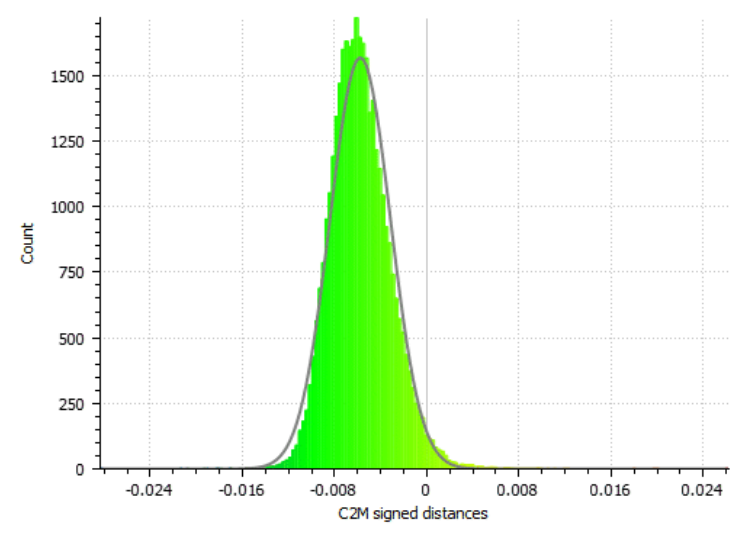

Gauss: mean $=-0.006927 /$ std.dev. $=0.006914$ [235 classes]

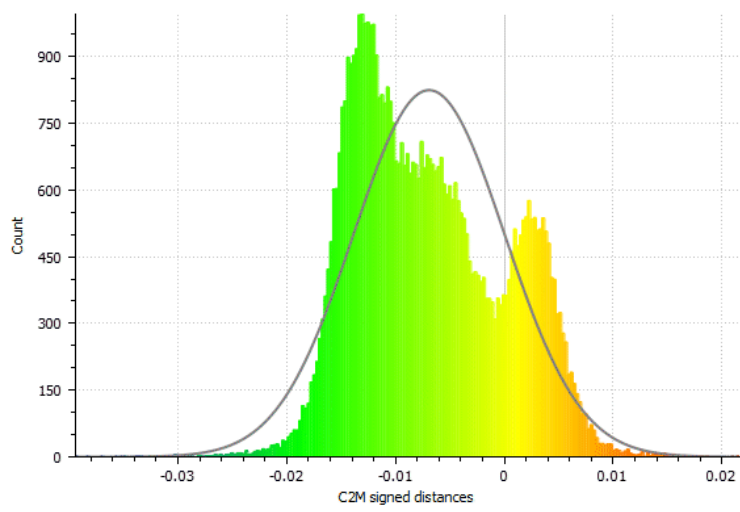

Figure 14. Comparison of the point cloud calculated with RealityCapture (BLK360 and images) compared to Faro

The advantage of laser scanning is the reliable capture of point clouds without the need for special engineering know-how. The prerequisites are stable positions and sufficient mutual overlaps or control point configurations. The time required for the recording in the field was $2-5$ times that of a photogrammetric image recording with the devices used. The Leica BLK360 laserscanner has proven to be a suitable device that delivers reliable results within its specifications.

The UAV-supported photogrammetry allows the measurement of roof and tower areas that cannot be detected by TLS. The use of even a simple, inexpensive drone has proven to be practical here. The terrestrial images can then be evaluated without any problems if a large overlap (approx. 80-90\%) is ensured and if 
in between there is no need for edged or out-of-line images. The evaluation of individual terrestrial images was not easily possible, but in combination with the UAV images all images could be oriented. The overall accuracy of approx. $5 \mathrm{~mm}$ is in the range of laser scanning. Depending on the hardware and software equipment, the required computing times may not be practicable due to the high number of images. However, RealityCapture's highly parallel solution shows that optimized implementations with simultaneous TLS data fusion are possible and lead to high-quality results.

Further investigations are planned for the future, in which more intensive analyses of the camera calibration and the dense point clouds will be carried out. It is also planned to test further SfM programs with these data.

\section{REFERENCES}

Adamopoulos, E., Tsilimantou, E., Keramidas, V., Apostolopoulou, M., Karoglou, M., Tapinaki, S., Ioannidis, C., Georgopoulos, A., Moropoulou, A., 2017. Multi-sensor documentation of metric and qualitative information of historic stone structures. Int. Arch. Photogramm. Remote Sens. Spatial Inf. Sci, IV-2/W2, 1-8.

Anchabadze, Y.D., Volkova, N.G., 1990. Altes Tbilisi. Miklucho-Maklai (eds.), Russian Academy of Sciences, Institute of Ethnography.

Beridze, V., 2014. The History of Georgian Architecture. Chubinashvili National Research Centre for History of Georgian Art and Monument Protection, Tumanishvili, D. (ed.), Tbilisi.

Blaskow, R., Lindstaedt, M., Schneider, D., Kersten, T., 2018. Untersuchungen zum Genauigkeitspotential des terrestrischen Laserscanners Leica BLK360. Photogrammetrie, Laserscanning, Optische 3D-Messtechnik - Beiträge der Oldenburger 3D-Tage 2018, Luhmann, T. \& Schumacher, C. (eds.), VDE Verlag GmbH, Berlin/Offenbach, 284-296.

Chizhova, M., Korovin, D., Brodowskii, M., Brunn, A., Stilla, U., Luhmann, T. (2017). Probabilistic reconstruction of orthodox churches from precision point clouds using cellular automata. 3D Arch 2017, Int. Arch. Photogramm. Remote Sens. Spatial Inf. Sci., XLII-2/W3, 187-194, doi: 10.5194/isprsarchives-XLII-2-W3-187-2017.

Kersten, T., Lindstaedt, M., Maziull, L., Schreyer, K., Tschirschwitz, F., Holm, K. (2015). 3D-Erfassung, Modellierung und Visualisierung der Festungsanlage Kristiansten in Trondheim durch Photogrammetrie und terrestrisches Laserscanning im Rahmen von ERASMUSProgrammen. Publikationen der DGPF, Band 24, T. Kersten (ed.), 78-89.

Luhmann, T., Chizhova, M., Gorkovchuk, D., Jepping, A., Hastedt, H., 2019. Kombination von terrestrischem Laserscanning, UAV- und Nahbereichsphotogrammetrie zur Erfassung komplexer Kirchenbauwerke in Georgien. Publikationen der DGPF, Band 28, T. Kersten (ed.), 27-43.

Remondino, F., 2011. Heritage Recording and 3D Modeling with Photogrammetry and 3D Scanning. Remote Sensing, 3(6), 1104-1138. 